\title{
TAILORED FINITE POINT METHOD FOR STEADY-STATE REACTION-DIFFUSION EQUATIONS*
}

\author{
HOUDE HAN ${ }^{\dagger}$ AND ZHONGYI HUANG
}

\begin{abstract}
In this paper, we propose to use the tailored-finite-point method (TFPM) for a type of steady-state reaction-diffusion problems in two dimensions. Three tailored finite point schemes are constructed for the given problem. Our finite point method has been tailored to some particular properties of the problem. Therefore, our TFPM satisfies the discrete maximum principle automatically. We also study the error estimates of our TFPM. We prove that our TFPM can achieve good accuracy even when the mesh size $h \gg \varepsilon$. Our numerical examples show the efficiency and reliability of our method.
\end{abstract}

Key words. Tailored finite point method, singular perturbation problem, boundary layer, discrete maximum principle.

AMS subject classifications. 65N30, 35J65.

\section{Introduction}

We consider the steady-state reaction-diffusion equation in the unit square $\Omega=$ $(0,1) \times(0,1)$ :

$$
\begin{aligned}
\mathbf{L} u \equiv-\varepsilon^{2} \triangle u+b(x, y) u & =f(x, y), \quad \text { in } \quad \Omega, \\
u & =0, \quad \text { on } \quad \Gamma=\partial \Omega,
\end{aligned}
$$

where $b(x, y)$ and $f(x, y)$ are two given functions on $\bar{\Omega}$ and

$$
b(x, y) \geq b_{\min }>0, \text { on } \bar{\Omega} .
$$

Furthermore we suppose that the given functions $b(x, y), f(x, y) \in \mathbf{C}^{4, \beta}(\bar{\Omega})$ for a real number $\beta \in(0,1)$, and the function $f(x, y)$ satisfies the corner compatibility conditions:

$$
f(0,0)=f(1,0)=f(0,1)=f(1,1)=0 .
$$

Then we know that the solution of problem $(1.1)-(1.2), u(x, y) \in \mathbf{C}^{6, \beta}(\Omega) \cap \mathbf{C}^{3, \beta}(\bar{\Omega})$ $[5]$.

The problem (1.1)-(1.2) is a singular perturbation problem when $\varepsilon \ll 1$; the solution of problem (1.1)-(1.2) is allowed boundary layers as well as corner layers [10]. These layers are characterized by rapid transitions in the solution, and are thus difficult to capture in a numerical approximation without using a large number of unknowns. Also, such layers tend to cause spurious oscillations in a numerical solution to the problem.

Methods for the numerical solution of problems such as (1.1)-(1.2) in bounded or unbounded domains that attempt to deal with these difficulties have been developed by many mathematicians, see e.g., $[2,3,12,14,15,16,18,19,20,22,23,24,25]$.

*Received: August 31, 2009; accepted (in revised version): December 22, 2009. Communicated by Tiejun Li. This work was partially supported by the NSFC Project No. 10971116, the NSAF Project No. 10676017, the National Basic Research Program of China under the grant 2005CB321701.

†Department of Mathematical Sciences, Tsinghua University, Beijing 100084, China (hhan@math. tsinghua.edu.cn).

‡Department of Mathematical Sciences, Tsinghua University, Beijing 100084, China (zhuang@ math.tsinghua.edu.cn). 
For one dimensional singular perturbation problems, people used the method of "exponential fitting" $[2,12,18,22]$ to get a uniform convergence. Recently, the typical methods for high dimensional problems are based on the adaptive mesh techniques, such as Shishkin meshes [3, 14, 15, 23], in which one can get uniform convergence on nonuniform meshes. Melenk et al also used the so-called $h p$-version finite element method to achieve uniform convergence (see [16] and the references in it). Generally speaking, they need the finest mesh size $h \sim \mathcal{O}(\varepsilon)$ to achieve a satisfactory numerical result.

In this paper, we propose using the tailored finite point method to deal with problem (1.1)-(1.2). The tailored finite point method [9] is a new discrete method for solving differential equations numerically. For each given problem, the discrete scheme has been tailored to some particular properties of the given problem. We have obtained good approximations using the tailored finite point method for solving singular perturbation problems [8,9], interface problems [11], and high frequency waves [7]. For problem (1.1)-(1.2), three tailored finite point schemes are constructed. Each of them can achieve high accuracy on uniform mesh with the mesh size $h \gg \varepsilon$.

The paper is organized as follows. In section 2 , we recall some asymptotic properties of the solution for problem (1.1)-(1.2). In section 3, we describe our tailored finite point method in details. We study the error analysis in section 4 . In section 5, we use some numerical examples to show the efficiency of our new method. Finally, we draw a conclusion in section 6 .

\section{Asymptotic properties of the analytic solution}

We now recall some properties of the solution $u(x, y)$ of problem (1.1)-(1.2) (cf. $[10,13]):$

THEOREM 2.1. The solution $u(x, y)$ of problem (1.1)-(1.2) is uniformly bounded on $\bar{\Omega}$, namely

$$
|u(x, y)| \leq M_{0},
$$

with

$$
M_{0}=\max _{(x, y) \in \bar{\Omega}} \frac{|f(x, y)|}{b(x, y)} .
$$

Proof. It is straightforward to prove it by maximum principle.

Theorem 2.2 (cf. Theorem 2.1 in [10]). Let $u(x, y)$ solve the problem (1.1)-(1.2), then the following asymptotic expansion holds:

$$
\begin{array}{r}
u(x, y)=U_{2}(x, y)+V_{2}(x, y)+W_{2}(x, y)+\tilde{V}_{2}(x, y)+\tilde{W}_{2}(x, y)+\sum_{j=1}^{4} Z_{2}^{j}(x, y)+R_{2}(x, y) \\
\forall(x, y) \in \bar{\Omega},
\end{array}
$$

where $U_{2}(x, y)$ is the outer expansion, $V_{2}(x, y), W_{2}(x, y), \tilde{V}_{2}(x, y)$, and $\tilde{W}_{2}(x, y)$ are the boundary layers, $\left\{Z_{2}^{j}(x, y),(j=1, \ldots, 4)\right\}$ are the corner layers, $R_{2}(x, y)$ is the remainder, and

$$
U_{2}(x, y)=\frac{f(x, y)}{b(x, y)}+\varepsilon^{2} \Delta\left(\frac{f(x, y)}{b(x, y)}\right)
$$




$$
\begin{aligned}
\left|V_{2}(x, y)\right| & \leq C e^{-\alpha \frac{x}{\varepsilon}} \\
\left|\tilde{V}_{2}(x, y)\right| & \leq C e^{-\alpha \frac{1-x}{\varepsilon}} \\
\left|W_{2}(x, y)\right| & \leq C e^{-\alpha \frac{y}{\varepsilon}} \\
\left|\tilde{W}_{2}(x, y)\right| & \leq C e^{-\alpha \frac{1-y}{\varepsilon}} \\
\left|Z_{2}^{1}(x, y)\right| & \leq C e^{-\alpha \frac{x+y}{\varepsilon}} \\
\left|Z_{2}^{2}(x, y)\right| & \leq C e^{-\alpha \frac{1-x+y}{\varepsilon}} \\
\left|Z_{2}^{3}(x, y)\right| & \leq C e^{-\alpha \frac{x+1-y}{\varepsilon}} \\
\left|Z_{2}^{4}(x, y)\right| & \leq C e^{-\alpha \frac{2-x-y}{\varepsilon}} \\
\left|R_{2}(x, y)\right| & \leq C \varepsilon^{3}
\end{aligned}
$$

with constants $C, \alpha$ independent of $\varepsilon$ and $\alpha \in\left(0, \sqrt{b_{\text {min }}}\right)$.

\section{The tailored finite point scheme}

In this section, we will explain how to construct our finite point scheme [7, 8, 9, 11], which is quite different from the typical finite point methods $[4,17,21]$. We call our new scheme a "tailored finite point method" (TFPM) because the finite point method has been tailored to some particular properties of the problem. The finite point method $[4,17,21]$ is a development of finite difference method in which the meshless technique is emphasized. For one dimensional singular perturbation problems, the method is very close to the method of "exponential fitting" discussed in $[2,12,18]$ and [22, Chapter I, section 2] .

Let $h=N^{-1}$ be the mesh size and

$$
x_{i}=i h, \quad y_{j}=j h, \quad 0 \leq i, j \leq N .
$$

Then $\left\{P_{i, j}=\left(x_{i}, y_{j}\right), \quad 0 \leq i, j \leq N\right\}$ are the mesh points and we have a uniform mesh.

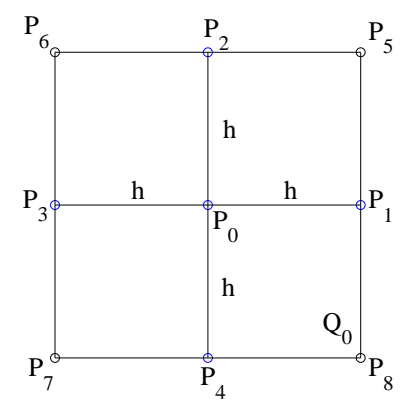

FIG. 3.1. The location of points $P_{0}, P_{1}, \cdots, P_{8}$.

For each mesh point $P_{0}$ in $\Omega$, there are four mesh points $\left\{P_{1}, P_{2}, P_{3}, P_{4}\right\}$ around $P_{0}$ and the distance between $P_{0}$ and $P_{i}(i=1, \ldots, 4)$ is $h$. Namely we obtain a cell $Q_{0}$ with center $P_{0}$ (see figure 3.1). We now construct the tailored finite point scheme of equation (1.1) at mesh point $P_{0}$. At first equation (1.1) is simplified in the cell $Q_{0}$ as

$$
-\varepsilon^{2} \triangle u+d_{0}^{2} u=f_{0}, \quad \text { in } Q_{0},
$$


where

$$
d_{0}^{2}=b\left(P_{0}\right), \quad f_{0}=f\left(P_{0}\right) .
$$

For any solution $u(x, y)$ of equation (3.2), let

$$
v(x, y)=u(x, y)-\frac{f_{0}}{d_{0}^{2}}, \quad \mu_{0}=\frac{d_{0}}{\varepsilon},
$$

then $v(x, y)$ satisfies:

$$
-\triangle v+\mu_{0}^{2} v=0, \quad \text { in } \quad Q_{0} .
$$

3.1. TFPM based on Bessel functions. The solution of equation (3.5), $v(x, y)$, can be expanded as the following:

$$
v(x, y)=\alpha_{0} I_{0}\left(\mu_{0} r\right)+\sum_{n=1}^{\infty} I_{n}\left(\mu_{0} r\right)\left[\alpha_{n} \cos n \theta+\beta_{n} \sin n \theta\right],
$$

with constants $\left\{\alpha_{0}, \quad \alpha_{n}, \beta_{n}, n=1,2, \ldots\right\}$.

We take

$$
v_{h}(x, y)=\alpha_{0} I_{0}\left(\mu_{0} r\right)+\alpha_{1} I_{1}\left(\mu_{0} r\right) \cos \theta+\beta_{1} I_{1}\left(\mu_{0} r\right) \sin \theta+\alpha_{2} I_{2}\left(\mu_{0} r\right) \cos 2 \theta .
$$

Let

$$
v_{h}\left(P_{j}\right)=V_{j}, \quad j=0,1,2,3,4,
$$

then we have

$$
\begin{aligned}
& V_{0}=\alpha_{0}, \\
& V_{1}=\alpha_{0} I_{0}\left(\mu_{0} r\right)+\alpha_{1} I_{1}\left(\mu_{0} r\right)+\alpha_{2} I_{2}\left(\mu_{0} r\right), \\
& V_{2}=\alpha_{0} I_{0}\left(\mu_{0} r\right)+\beta_{1} I_{1}\left(\mu_{0} r\right)-\alpha_{2} I_{2}\left(\mu_{0} r\right), \\
& V_{3}=\alpha_{0} I_{0}\left(\mu_{0} r\right)-\alpha_{1} I_{1}\left(\mu_{0} r\right)+\alpha_{2} I_{2}\left(\mu_{0} r\right), \\
& V_{4}=\alpha_{0} I_{0}\left(\mu_{0} r\right)-\beta_{1} I_{1}\left(\mu_{0} r\right)-\alpha_{2} I_{2}\left(\mu_{0} r\right) .
\end{aligned}
$$

Namely we have

$$
\left(\begin{array}{l}
V_{1} \\
V_{2} \\
V_{3} \\
V_{4}
\end{array}\right)=\mathbf{A}\left(\begin{array}{c}
\alpha_{0} \\
\alpha_{1} \\
\beta_{1} \\
\alpha_{2}
\end{array}\right)
$$

with

$$
\mathbf{A}=\left(\begin{array}{cccr}
I_{0}\left(\mu_{0} h\right) & I_{1}\left(\mu_{0} h\right) & 0 & I_{2}\left(\mu_{0} h\right) \\
I_{0}\left(\mu_{0} h\right) & 0 & I_{1}\left(\mu_{0} h\right) & -I_{2}\left(\mu_{0} h\right) \\
I_{0}\left(\mu_{0} h\right) & -I_{1}\left(\mu_{0} h\right) & 0 & I_{2}\left(\mu_{0} h\right) \\
I_{0}\left(\mu_{0} h\right) & 0 & -I_{1}\left(\mu_{0} h\right)-I_{2}\left(\mu_{0} h\right)
\end{array}\right)
$$

The matrix $\mathbf{A}$ is invertible and

$$
\mathbf{A}^{-1}=\left(\begin{array}{cccc}
\frac{1}{4 I_{0}\left(\mu_{0} h\right)} & \frac{1}{4 I_{0}\left(\mu_{0} h\right)} & \frac{1}{4 I_{0}\left(\mu_{0} h\right)} & \frac{1}{4 I_{0}\left(\mu_{0} h\right)} \\
\frac{1}{2 I_{1}\left(\mu_{0} h\right)} & 0 & -\frac{1}{2 I_{1}\left(\mu_{0} h\right)} & 0 \\
0 & \frac{1}{2 I_{1}\left(\mu_{0} h\right)} & 0 & -\frac{1}{2 I_{1}\left(\mu_{0} h\right)} \\
\frac{1}{4 I_{2}\left(\mu_{0} h\right)} & -\frac{1}{4 I_{2}\left(\mu_{0} h\right)} & \frac{1}{4 I_{2}\left(\mu_{0} h\right)} & -\frac{1}{4 I_{2}\left(\mu_{0} h\right)}
\end{array}\right)
$$




$$
\left(\begin{array}{l}
\alpha_{0} \\
\alpha_{1} \\
\beta_{1} \\
\alpha_{2}
\end{array}\right)=\mathbf{A}^{-1}\left(\begin{array}{l}
V_{1} \\
V_{2} \\
V_{3} \\
V_{4}
\end{array}\right) .
$$

From the equalities (3.9) and (3.17), we arrive at

$$
V_{0}-\frac{1}{4 I_{0}\left(\mu_{0} h\right)}\left(V_{1}+V_{2}+V_{3}+V_{4}\right)=0 .
$$

This is a 5-point discrete scheme for equation (3.5). From equality (3.4), let

$$
U_{j}=V_{j}+\frac{f_{0}}{d_{0}^{2}}, \quad j=0,1, \ldots, 4,
$$

then we obtain

$$
U_{0}-\frac{1}{4 I_{0}\left(\mu_{0} h\right)}\left(U_{1}+U_{2}+U_{3}+U_{4}\right)=\left(1-\frac{1}{I_{0}\left(\mu_{0} h\right)}\right) \frac{f_{0}}{d_{0}^{2}} .
$$

Namely,

$$
\left(\mathbf{L}_{h} U\right)\left(P_{0}\right) \equiv \frac{d_{0}^{2} I_{0}\left(\mu_{0} h\right)}{I_{0}\left(\mu_{0} h\right)-1}\left\{U_{0}-\frac{1}{4 I_{0}\left(\mu_{0} h\right)}\left(U_{1}+U_{2}+U_{3}+U_{4}\right)\right\}=f_{0} .
$$

The discrete scheme (3.21) is called the 5-point tailored finite point scheme of equation (1.1).

For the case $0<\mu_{0} h \ll 1$, we know that $[1,6]$

$$
I_{0}\left(\mu_{0} h\right)=1+\frac{1}{4}\left(\mu_{0} h\right)^{2}+\mathcal{O}\left(\mu_{0} h\right)^{4} .
$$

Using $1+\frac{1}{4}\left(\mu_{0} h\right)^{2}$ to approximate $I_{0}\left(\mu_{0} h\right)$, then the tailored finite point scheme (3.21) is reduced to the following standard second-order finite difference scheme of equation (1.1),

$$
-\varepsilon^{2} \frac{U_{1}+U_{2}+U_{3}+U_{4}-4 U_{0}}{h^{2}}+d_{0}^{2} U_{0}=f_{0} .
$$

3.2. TFPM based on exponential functions. We can also construct a scheme for (3.5) as follows. Let

$$
H_{4}=\left\{v(x, y) \mid v=c_{1} e^{-\mu_{0} x}+c_{2} e^{\mu_{0} x}+c_{3} e^{-\mu_{0} y}+c_{4} e^{\mu_{0} y}, \quad \forall c_{1}, c_{2}, c_{3}, c_{4} \in \mathbb{R}\right\} .
$$

It is straightforward to check that every function in the space $H_{4}$ is a solution of equation (3.5). Then we take a scheme as

$$
\alpha_{1} v_{1}+\alpha_{2} v_{2}+\alpha_{3} v_{3}+\alpha_{4} v_{4}+\alpha_{0} v_{0}=0,
$$

with $v_{i}=v\left(P_{i}\right)$, such that (3.24) holds for all $v \in H_{4}$. Thus we obtain

$$
\begin{aligned}
& \alpha_{1} e^{-\mu_{0} h}+\alpha_{2}+\alpha_{3} e^{\mu_{0} h}+\alpha_{4}+\alpha_{0}=0, \\
& \alpha_{1} e^{\mu_{0} h}+\alpha_{2}+\alpha_{3} e^{-\mu_{0} h}+\alpha_{4}+\alpha_{0}=0, \\
& \alpha_{1}+\alpha_{2} e^{-\mu_{0} h}+\alpha_{3}+\alpha_{4} e^{\mu_{0} h}+\alpha_{0}=0, \\
& \alpha_{1}+\alpha_{2} e^{\mu_{0} h}+\alpha_{3}+\alpha_{4} e^{-\mu_{0} h}+\alpha_{0}=0
\end{aligned}
$$


That means, for any given $\alpha_{0} \in \mathbb{R}$, the system (3.25)-(3.28) has the unique solution

$$
\alpha_{1}=\alpha_{2}=\alpha_{3}=\alpha_{4}=\frac{-\alpha_{0}}{e^{\mu_{0} h}+e^{-\mu_{0} h}+2} \equiv \frac{-\alpha_{0}}{4 \cosh ^{2}\left(\frac{\mu_{0} h}{2}\right)} .
$$

If we take

$$
\alpha_{0}=\frac{e^{\mu_{0} h}+e^{-\mu_{0} h}+2}{e^{\mu_{0} h}+e^{-\mu_{0} h}-2} \equiv \frac{\cosh ^{2}\left(\frac{\mu_{0} h}{2}\right)}{\sinh ^{2}\left(\frac{\mu_{0} h}{2}\right)},
$$

we obtain

$$
\alpha_{1}=\alpha_{2}=\alpha_{3}=\alpha_{4}=-\frac{1}{e^{\mu_{0} h}+e^{-\mu_{0} h}-2} \equiv-\frac{1}{4 \sinh ^{2}\left(\frac{\mu_{0} h}{2}\right)} .
$$

We finally have the following five-point scheme for (3.2):

$$
-\frac{d_{0}^{2}}{4\left(\cosh ^{2}\left(\frac{\mu_{0} h}{2}\right)-1\right)}\left(u_{1}+u_{2}+u_{3}+u_{4}-4 u_{0}\right)+d_{0}^{2} u_{0}=f_{0} .
$$

REMARK 3.1. If $h \ll \varepsilon$, i.e. $\mu_{0} h \ll 1$, we have

$$
4\left(\cosh ^{2}\left(\frac{\mu_{0} h}{2}\right)-1\right)=\left(\mu_{0} h\right)^{2}+\mathcal{O}\left(\left(\mu_{0} h\right)^{4}\right) .
$$

If we omit the high order term, the tailored finite point scheme (3.32) is also reduced to the standard second-order finite difference scheme (3.23) of equation (1.1).

Certainly, we can also construct a nine-point scheme for (3.5) as follows. Let

$$
\begin{gathered}
H_{8}=\left\{v(x, y) \mid v=c_{1} e^{-\mu_{0} x}+c_{2} e^{\mu_{0} x}+c_{3} e^{-\mu_{0} y}+c_{4} e^{\mu_{0} y}+c_{5} e^{\frac{\mu_{0}(x+y)}{\sqrt{2}}}+c_{6} e^{-\frac{\mu_{0}(x+y)}{\sqrt{2}}}\right. \\
\left.+c_{7} e^{\frac{\mu_{0}(x-y)}{\sqrt{2}}}+c_{8} e^{-\frac{\mu_{0}(x-y)}{\sqrt{2}}}, \quad \forall c_{j} \in \mathbb{R}, j=1, \cdots, 8\right\} .
\end{gathered}
$$

It is also easy to check that all the functions in the space $H_{8}$ is a solution of equation (3.5). Then we take a scheme as

$$
\alpha_{1} v_{1}+\alpha_{2} v_{2}+\alpha_{3} v_{3}+\alpha_{4} v_{4}+\alpha_{5} v_{5}+\alpha_{6} v_{6}+\alpha_{7} v_{7}+\alpha_{8} v_{8}+\alpha_{0} v_{0}=0
$$

with $v_{i}=v\left(P_{i}\right)$, such that (3.33) holds for all $v \in H_{8}$. Similarly, we obtain

$$
\begin{array}{r}
\alpha_{1} e^{-\mu_{0} h}+\alpha_{2}+\alpha_{3} e^{\mu_{0} h}+\alpha_{4}+\alpha_{5} e^{-\mu_{0} h}+\alpha_{6} e^{\mu_{0} h}+\alpha_{7} e^{\mu_{0} h}+\alpha_{8} e^{-\mu_{0} h}+\alpha_{0}=0, \\
\alpha_{1} e^{\mu_{0} h}+\alpha_{2}+\alpha_{3} e^{-\mu_{0} h}+\alpha_{4}+\alpha_{5} e^{\mu_{0} h}+\alpha_{6} e^{-\mu_{0} h}+\alpha_{7} e^{-\mu_{0} h}+\alpha_{8} e^{\mu_{0} h}+\alpha_{0}=0, \\
\alpha_{1}+\alpha_{2} e^{-\mu_{0} h}+\alpha_{3}+\alpha_{4} e^{\mu_{0} h}+\alpha_{5} e^{-\mu_{0} h}+\alpha_{6} e^{-\mu_{0} h}+\alpha_{7} e^{\mu_{0} h}+\alpha_{8} e^{\mu_{0} h}+\alpha_{0}=0, \\
\alpha_{1}+\alpha_{2} e^{\mu_{0} h}+\alpha_{3}+\alpha_{4} e^{-\mu_{0} h}+\alpha_{5} e^{\mu_{0} h}+\alpha_{6} e^{\mu_{0} h}+\alpha_{7} e^{-\mu_{0} h}+\alpha_{8} e^{-\mu_{0} h}+\alpha_{0}=0, \\
\alpha_{1} e^{\frac{\mu_{0} h}{\sqrt{2}}}+\alpha_{2} e^{\frac{\mu_{0} h}{\sqrt{2}}}+\alpha_{3} e^{-\frac{\mu_{0} h}{\sqrt{2}}}+\alpha_{4} e^{-\frac{\mu_{0} h}{\sqrt{2}}}+\alpha_{5} e^{\sqrt{2} \mu_{0} h}+\alpha_{6}+\alpha_{7} e^{-\sqrt{2} \mu_{0} h}+\alpha_{8}+\alpha_{0}=0, \\
\alpha_{1} e^{-\frac{\mu_{0} h}{\sqrt{2}}}+\alpha_{2} e^{-\frac{\mu_{0} h}{\sqrt{2}}}+\alpha_{3} e^{\frac{\mu_{0} h}{\sqrt{2}}}+\alpha_{4} e^{\frac{\mu_{0} h}{\sqrt{2}}}+\alpha_{5} e^{-\sqrt{2} \mu_{0} h}+\alpha_{6}+\alpha_{7} e^{\sqrt{2} \mu_{0} h}+\alpha_{8}+\alpha_{0}=0, \\
\alpha_{1} e^{\frac{\mu_{0} h}{\sqrt{2}}}+\alpha_{2} e^{-\frac{\mu_{0} h}{\sqrt{2}}}+\alpha_{3} e^{-\frac{\mu_{0} h}{\sqrt{2}}}+\alpha_{4} e^{\frac{\mu_{0} h}{\sqrt{2}}}+\alpha_{5}+\alpha_{6} e^{-\sqrt{2} \mu_{0} h}+\alpha_{7}+\alpha_{8} e^{\sqrt{2} \mu_{0} h}+\alpha_{0}=0, \\
\alpha_{1} e^{-\frac{\mu_{0} h}{\sqrt{2}}}+\alpha_{2} e^{\frac{\mu_{0} h}{\sqrt{2}}}+\alpha_{3} e^{\frac{\mu_{0} h}{\sqrt{2}}}+\alpha_{4} e^{-\frac{\mu_{0} h}{\sqrt{2}}}+\alpha_{5}+\alpha_{6} e^{\sqrt{2} \mu_{0} h}+\alpha_{7}+\alpha_{8} e^{-\sqrt{2} \mu_{0} h}+\alpha_{0}=0 .
\end{array}
$$


Therefore, for any given $\alpha_{0} \in \mathbb{R}$, the system (3.34)-(3.34) has the unique solution

$$
\begin{aligned}
& \alpha_{1}=\alpha_{2}=\alpha_{3}=\alpha_{4}=\frac{-\alpha_{0}\left(\cosh ^{2}\left(\frac{\mu_{0} h}{\sqrt{2}}\right)-\cosh \left(\mu_{0} h\right)\right)}{4\left(\cosh ^{2}\left(\frac{\mu_{0} h}{2}\right) \cosh ^{2}\left(\frac{\mu_{0} h}{\sqrt{2}}\right)-\cosh \left(\mu_{0} h\right) \cosh \left(\frac{\mu_{0} h}{\sqrt{2}}\right)\right)}, \\
& \alpha_{5}=\alpha_{6}=\alpha_{7}=\alpha_{8}=\frac{-\alpha_{0}\left(\cosh ^{2}\left(\frac{\mu_{0} h}{2}\right)-\cosh \left(\frac{\mu_{0} h}{\sqrt{2}}\right)\right)}{4\left(\cosh ^{2}\left(\frac{\mu_{0} h}{2}\right) \cosh ^{2}\left(\frac{\mu_{0} h}{\sqrt{2}}\right)-\cosh \left(\mu_{0} h\right) \cosh \left(\frac{\mu_{0} h}{\sqrt{2}}\right)\right)} .
\end{aligned}
$$

If we take

$$
\alpha_{0}=4\left(\cosh ^{2}\left(\frac{\mu_{0} h}{2}\right) \cosh ^{2}\left(\frac{\mu_{0} h}{\sqrt{2}}\right)-\cosh \left(\mu_{0} h\right) \cosh \left(\frac{\mu_{0} h}{\sqrt{2}}\right)\right),
$$

we have

$$
\begin{aligned}
& \alpha_{1}=\alpha_{2}=\alpha_{3}=\alpha_{4}=\cosh \left(\mu_{0} h\right)-\cosh ^{2}\left(\frac{\mu_{0} h}{\sqrt{2}}\right), \\
& \alpha_{5}=\alpha_{6}=\alpha_{7}=\alpha_{8}=\cosh \left(\frac{\mu_{0} h}{\sqrt{2}}\right)-\cosh ^{2}\left(\frac{\mu_{0} h}{2}\right) .
\end{aligned}
$$

From the expression (3.36)-(3.38), we know that $\forall h>0$,

$$
\alpha_{0}>0, \quad \alpha_{1}<0, \quad \alpha_{5}<0 ; \quad \alpha_{0}+4\left(\alpha_{1}+\alpha_{5}\right)>0 .
$$

We finally get the following nine-point scheme for (3.2):

$$
d_{0}^{2} \frac{\alpha_{1}\left(u_{1}+u_{2}+u_{3}+u_{4}-4 u_{0}\right)+\alpha_{5}\left(u_{5}+u_{6}+u_{7}+u_{8}-4 u_{0}\right)}{\alpha_{0}+4\left(\alpha_{1}+\alpha_{5}\right)}+d_{0}^{2} u_{0}=f_{0} .
$$

REMARK 3.2. It is easy to check that all of our tailored finite point schemes, i.e. (3.20), (3.32), and (3.40), satisfy the discrete maximum principle. Because $\mu_{0} h>$ 0 , we have $I_{0}\left(\mu_{0} h\right)>1$ and $\cosh \left(\frac{\mu_{0} h}{2}\right)>1$. It is straightforward to check that schemes (3.20) and (3.32) are diagonally dominant. Because of (3.39), we know that scheme (3.40) is also diagonally dominant.

\section{Error analysis}

4.1. Truncation error. We now consider the truncation error of schemes (3.21), (3.32), and (3.40) at mesh point $P_{0} \in \Omega$.

4.1.1. Truncation error for (3.21). For a function $u$, the solution of problem (1.1)-(1.2), we have

$$
\begin{aligned}
\left(\mathbf{L}_{h} u\right)\left(P_{0}\right) & =\frac{d_{0}^{2} I_{0}\left(\mu_{0} h\right)}{I_{0}\left(\mu_{0} h\right)-1}\left\{u_{0}-\frac{1}{4 I_{0}\left(\mu_{0} h\right)}\left(u_{1}+u_{2}+u_{3}+u_{4}\right)\right\} \\
& =d_{0}^{2} u_{0}-\frac{d_{0}^{2}}{4\left(I_{0}\left(\mu_{0} h\right)-1\right)}\left(u_{1}+u_{2}+u_{3}+u_{4}-4 u_{0}\right), \\
(\mathbf{L} u)\left(P_{0}\right) & =f_{0}
\end{aligned}
$$

with

$$
u_{j}=u\left(P_{j}\right), \quad j=0,1, \ldots, 4 .
$$


The truncation error of scheme (3.21) at mesh point $P_{0}$ is given as

$$
\begin{aligned}
\left(\mathbf{T}_{h} u\right)\left(P_{0}\right) & =\left(\mathbf{L}_{h} u\right)\left(P_{0}\right)-(\mathbf{L} u)\left(P_{0}\right) \\
& =d_{0}^{2} u_{0}-f_{0}-\frac{d_{0}^{2}}{4\left(I_{0}\left(\mu_{0} h\right)-1\right)}\left(u_{1}+u_{2}+u_{3}+u_{4}-4 u_{0}\right) .
\end{aligned}
$$

Is the truncation error $\left(\mathbf{T}_{h} u\right)\left(P_{0}\right)$ small? In the case $\varepsilon \ll h<1$, the answer is 'yes'. More precisely, we consider the case

$$
\frac{\varepsilon}{h} \leq h^{\gamma_{0}},
$$

where $\gamma_{0}>0$ is a constant. It means that we used a coarse mesh with respect to the small parameter $\varepsilon$. We now estimate the truncation error at point $P_{0}=\left(x_{0}, y_{0}\right)$. Since $P_{0}$ is a mesh point in $\Omega$, we know that

$$
h \leq x_{0} \leq 1-h, \quad h \leq y_{0} \leq 1-h .
$$

By the expansion (2.3), we have

$$
\begin{aligned}
d_{0}^{2} u\left(P_{0}\right)-f_{0}=d_{0}^{2}\{ & \varepsilon^{2} \Delta\left(\frac{f}{b}\right)\left(P_{0}\right)+V_{2}\left(P_{0}\right)+W_{2}\left(P_{0}\right) \\
& \left.+\tilde{V}_{2}\left(P_{0}\right)+\tilde{W}_{2}\left(P_{0}\right)+\sum_{j=1}^{4} Z_{2}^{j}\left(P_{0}\right)+R_{2}\left(P_{0}\right)\right\} .
\end{aligned}
$$

Combining condition (4.4) and estimates (2.4)-(2.13), we obtain

$$
\left|d_{0}^{2} u\left(P_{0}\right)-f_{0}\right| \leq C\left\{h^{2+2 \gamma_{0}}+e^{-\alpha \frac{h}{\varepsilon}}\right\} .
$$

On the other hand, $u(x, y)$, the solution of problem (1.1)-(1.2) is uniformly bounded on $\bar{\Omega}$ (see (2.1)), then we have

$$
\left|\frac{d_{0}^{2}}{I_{0}\left(d_{0} \frac{h}{\varepsilon}\right)-1}\left(u_{1}+u_{2}+u_{3}+u_{4}-4 u_{0}\right)\right| \leq \frac{8 M_{0} d_{0}^{2}}{I_{0}\left(d_{0} \frac{h}{\varepsilon}\right)-1} \leq C e^{-\alpha \frac{h}{\varepsilon}} .
$$

Finally we arrive at

$$
\left|\left(\mathbf{T}_{h} u\right)\left(P_{0}\right)\right| \leq C\left\{h^{2+2 \gamma_{0}}+e^{-\alpha\left(h^{-\gamma_{0}}\right)}\right\} .
$$

4.1.2. Truncation error for (3.32) and (3.40). Similarly, the truncation error of scheme (3.32) at mesh point $P_{0}$ is given by

$$
\begin{aligned}
\left(\mathbf{T}_{h} u\right)\left(P_{0}\right) & =\left(\mathbf{L}_{h} u\right)\left(P_{0}\right)-(\mathbf{L} u)\left(P_{0}\right) \\
& =d_{0}^{2} u_{0}-f_{0}-\frac{d_{0}^{2}}{4\left(\cosh ^{2}\left(\frac{\mu_{0} h}{2}\right)-1\right)}\left(u_{1}+u_{2}+u_{3}+u_{4}-4 u_{0}\right) .
\end{aligned}
$$

Also because the solution of problem (1.1)-(1.2) is uniformly bounded on $\bar{\Omega}$, we have

$$
\left|\frac{d_{0}^{2}}{4\left(\cosh ^{2}\left(\frac{\mu_{0} h}{2}\right)-1\right)}\left(u_{1}+u_{2}+u_{3}+u_{4}-4 u_{0}\right)\right| \leq \frac{2 M_{0} d_{0}^{2}}{\cosh ^{2}\left(\frac{d_{0} h}{2 \varepsilon}\right)-1} \leq C e^{-\alpha \frac{h}{\varepsilon}} .
$$


Finally we also get the truncation error for (3.32) as

$$
\left|\left(\mathbf{T}_{h} u\right)\left(P_{0}\right)\right| \leq C\left\{h^{2+2 \gamma_{0}}+e^{-\alpha\left(h^{-\gamma_{0}}\right)}\right\} .
$$

For the truncation error of scheme (3.37), because

$$
\alpha_{0} \sim e^{(1+\sqrt{2}) \mu_{0} h}, \quad \alpha_{1} \sim e^{\sqrt{2} \mu_{0} h}, \quad \alpha_{5} \sim e^{\mu_{0} h}, \quad \text { for } \mu_{0} h \gg 1,
$$

we can also get the truncation error for (3.37) as

$$
\left|\left(\mathbf{T}_{h} u\right)\left(P_{0}\right)\right| \leq C\left\{h^{2+2 \gamma_{0}}+e^{-\alpha\left(h^{-\gamma_{0}}\right)}\right\} .
$$

4.2. Error estimate. We now consider the discrete scheme of problem (1.1)(1.2) on the mesh (3.1), where $h$ satisfies condition (4.4).

4.2.1. Error estimate for scheme (3.21). For scheme (3.21), using the notation in (3.1), we have

$$
\begin{gathered}
\mathbf{L}_{h} U_{i, j} \equiv \frac{d_{i, j}^{2} I_{0}\left(d_{i, j} \frac{h}{\varepsilon}\right)}{I_{0}\left(d_{i, j} \frac{h}{\varepsilon}\right)-1}\left\{U_{i, j}-\frac{1}{4 I_{0}\left(d_{i, j} \frac{h}{\varepsilon}\right)}\left(U_{i+1, j}+U_{i, j+1}+U_{i-1, j}+U_{i, j-1}\right)\right\}=f_{i, j} \\
1 \leq i, j \leq N-1 \\
U_{i, 0}=U_{i, N}=0, \quad i=0,1, \ldots, N \\
U_{0, j}=U_{N, j}=0, \quad j=1, \ldots, N-1 .
\end{gathered}
$$

For the discrete problem (4.14)-(4.16), we have the following result:

TheOREM 4.1. Suppose that $\left\{U_{i, j}, \quad 0 \leq i, j \leq N\right\}$ is a solution of problem (4.14)(4.16). Then the following estimate holds:

$$
\left|U_{i, j}\right| \leq \max _{0 \leq i, j \leq N} \frac{\left|f_{i, j}\right|}{d_{i, j}^{2}}, \quad 0 \leq i, j \leq N .
$$

Proof.

(1) Suppose that there exists a mesh point $P_{i_{0}, j_{0}}$ such that for $U_{i_{0}, j_{0}}>0$ and

$$
U_{i, j} \leq U_{i_{0}, j_{0}}, \quad 0 \leq i, j \leq N .
$$

By the boundary conditions, we know that $1 \leq i_{0}, j_{0} \leq N-1$, from the discrete equation (4.14) we obtain

$$
U_{i_{0}, j_{0}} \leq \frac{f_{i_{0}, j_{0}}}{d_{i_{0}, j_{0}}^{2}}
$$

Namely we have:

$$
U_{i, j} \leq U_{i_{0}, j_{0}} \leq \frac{f_{i_{0}, j_{0}}}{d_{i_{0}, j_{0}}^{2}} \leq \max _{0 \leq i, j \leq N} \frac{\left|f_{i, j}\right|}{d_{i, j}^{2}}, \quad 0 \leq i, j \leq N .
$$

(2) On the other hand, if the hypothesis in (1) is not true, namely

$$
U_{i, j} \leq 0, \quad 0 \leq i, j \leq N .
$$


Combining inequalities (4.20)-(4.21) we obtain the upper bound estimate

$$
U_{i, j} \leq \max _{0 \leq i, j \leq N} \frac{\left|f_{i, j}\right|}{d_{i, j}^{2}}, \quad 0 \leq i, j \leq N,
$$

for $\left\{U_{i, j}, 0 \leq i, j \leq N\right\}$, the solution of problem (4.10)-(4.12).

Similarly for $\left\{U_{i, j}, \quad 0 \leq i, j \leq N\right\}$, the solution of problem (4.10)-(4.12), we can obtain the lower bound estimate

$$
U_{i, j} \geq-\max _{0 \leq i, j \leq N} \frac{\left|f_{i, j}\right|}{d_{i, j}^{2}}, \quad 0 \leq i, j \leq N
$$

Theorem 4.1 is proved completely.

Let

$$
E_{i, j}=U_{i, j}-u\left(P_{i, j}\right), \quad 0 \leq i, j \leq N
$$

then

$$
\mathbf{L}_{h} E=\mathbf{T}_{h} u=\mathcal{O}\left\{h^{2+2 \gamma_{0}}+e^{-\alpha h^{-\gamma_{0}}}\right\} .
$$

By Theorem 4.1, we have:

THEOREM 4.2. Under Assumption (4.4), the following error estimate holds:

$$
\left|E_{i, j}\right|=C\left\{h^{2+2 \gamma_{0}}+e^{-\alpha h^{-\gamma_{0}}}\right\}, \quad 0 \leq i, j \leq N
$$

with a constant $C$ independent of $\varepsilon$ and $h$.

4.2.2. Error estimate for schemes (3.32) and (3.37).

Similarly, we have the following theorem for schemes (3.32) and (3.37):

TheOREM 4.3. Under Assumption (4.4), the following error estimate holds:

$$
\left|E_{i, j}\right|=C\left\{h^{2+2 \gamma_{0}}+e^{-\alpha h^{-\gamma_{0}}}\right\}, \quad 0 \leq i, j \leq N
$$

with a constant $C$ independent of $\varepsilon$ and $h$.

\section{Numerical examples}

In this section, we will use some examples to show the accuracy and efficiency of our method.

EXAMPLE 5.1. First, we consider the following problem:

$$
\begin{aligned}
& -\varepsilon^{2} \triangle u+b(\mathbf{x}) u=f(\mathbf{x}), \quad \forall \mathbf{x}=(x, y) \in \Omega, \\
& \left.u\right|_{\partial \Omega}=0
\end{aligned}
$$

where

$$
\Omega=[0,1]^{2}, \quad b(\mathbf{x})=1+x+y, \quad f(\mathbf{x})=\sin \pi(x+y) .
$$

We denote $u^{e x}$ to be the 'exact' solution which we get by very fine mesh, $u^{1}$ to be the approximate solution which we get by scheme $(3.21), u^{2}$ to be the approximate 


\begin{tabular}{c|cccc}
\multicolumn{5}{c}{$\varepsilon=10^{-3}$} \\
\hline $\operatorname{mesh}$ size $h$ & $1 / 4$ & $1 / 8$ & $1 / 16$ & $1 / 32$ \\
\hline $\max _{i j}\left|E_{i j}^{1}\right|$ & $9.02 \mathrm{E}-8$ & $3.27 \mathrm{E}-7$ & $4.87 \mathrm{E}-7$ & $5.04 \mathrm{E}-7$ \\
\hline $\max _{i j}\left|E_{i j}^{2}\right|$ & $9.02 \mathrm{E}-8$ & $3.27 \mathrm{E}-7$ & $4.87 \mathrm{E}-7$ & $5.04 \mathrm{E}-7$ \\
\hline $\max _{i j}\left|E_{i j}^{3}\right|$ & $9.02 \mathrm{E}-8$ & $3.27 \mathrm{E}-7$ & $4.87 \mathrm{E}-7$ & $5.04 \mathrm{E}-7$ \\
\hline
\end{tabular}

\begin{tabular}{c|cccc}
\multicolumn{5}{c}{$\varepsilon=10^{-6}$} \\
\hline mesh size $h$ & $1 / 4$ & $1 / 8$ & $1 / 16$ & $1 / 32$ \\
\hline $\max _{i j}\left|E_{i j}^{1}\right|$ & 0.0 & 0.0 & 0.0 & 0.0 \\
\hline $\max _{i j}\left|E_{i j}^{2}\right|$ & 0.0 & 0.0 & 0.0 & 0.0 \\
\hline $\max _{i j}\left|E_{i j}^{3}\right|$ & 0.0 & 0.0 & 0.0 & 0.0 \\
\hline
\end{tabular}

TABLE 5.1. $L^{\infty}$ errors of the numerical solutions for different mesh size.
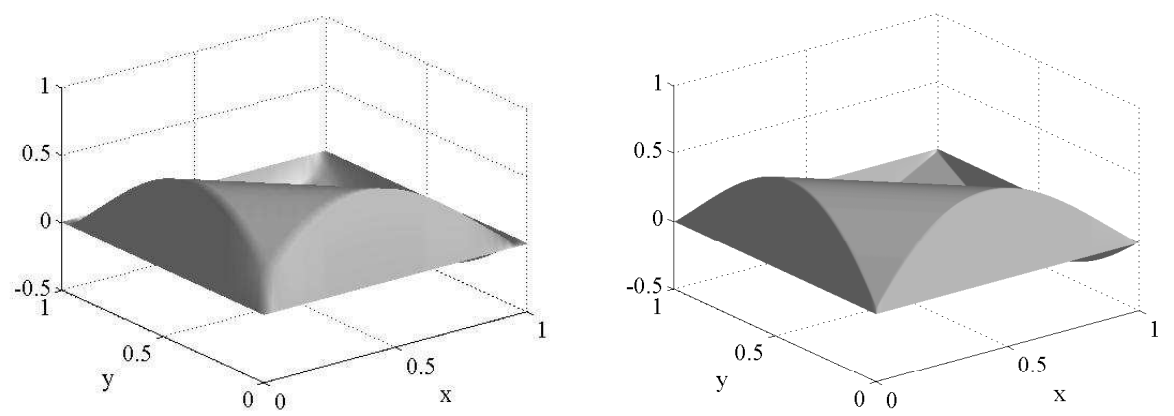

FIG. 5.1. The graphs of $u^{1}$ for Example 5.1, $\varepsilon=10^{-3}: h=\frac{1}{32}$ (left), $h=\frac{1}{128}$ (right).

solution which we get by scheme (3.32), and $u^{3}$ to be the approximate solution which we get by scheme (3.40). Then we let

$$
E^{1}=u^{1}-u^{e x}, \quad E^{2}=u^{2}-u^{e x}, \quad E^{3}=u^{3}-u^{e x} .
$$

The numerical results of example 5.1 are shown in Table 5.1 and figure 5.1.

EXAMPLE 5.2. Then, we consider the problem (5.1)-(5.2) with different choice of coefficients:

$$
b(\mathbf{x})=1+(x-0.5)^{2}+(y-0.5)^{2}, \quad f(\mathbf{x})=(x y(1-x)(1-y))^{\frac{1}{10}} .
$$

The numerical results of Example 5.2 are shown in Table 5.2 and figure 5.2.

From Tables 5.1-5.2, we can see that all of our schemes achieve very high accuracy for $h \gg \varepsilon$, which is consistent with the error estimates (4.26)-(4.27). Our schemes can achieve machine accuracy for $\varepsilon=10^{-6}$. From the error analysis, we know that the difference between our three schemes is mainly the coefficient on the exponential term. Then the errors in Tables 5.1-5.2 are almost the same for three schemes because $h \gg \epsilon$. It is clear that our methods can capture the boundary layers very accurately without any spurious oscillations on coarse meshes (see figures 5.1-5.2). 


\begin{tabular}{c|cccc}
\multicolumn{5}{c}{$\varepsilon=10^{-3}$} \\
\hline mesh size $h$ & $1 / 4$ & $1 / 8$ & $1 / 16$ & $1 / 32$ \\
\hline $\max _{i j}\left|E_{i j}^{1}\right|$ & $1.59 \mathrm{E}-6$ & $1.59 \mathrm{E}-6$ & $1.59 \mathrm{E}-6$ & $1.59 \mathrm{E}-6$ \\
\hline $\max _{i j}\left|E_{i j}^{2}\right|$ & $1.59 \mathrm{E}-6$ & $1.59 \mathrm{E}-6$ & $1.59 \mathrm{E}-6$ & $1.59 \mathrm{E}-6$ \\
\hline $\max _{i j}\left|E_{i j}^{3}\right|$ & $1.59 \mathrm{E}-6$ & $1.59 \mathrm{E}-6$ & $1.59 \mathrm{E}-6$ & $1.59 \mathrm{E}-6$ \\
\hline
\end{tabular}

\begin{tabular}{c|cccc}
\multicolumn{5}{c}{$\varepsilon=10^{-6}$} \\
\hline mesh size $h$ & $1 / 4$ & $1 / 8$ & $1 / 16$ & $1 / 32$ \\
\hline $\max _{i j}\left|E_{i j}^{1}\right|$ & 0.0 & 0.0 & 0.0 & 0.0 \\
\hline $\max _{i j}\left|E_{i j}^{2}\right|$ & 0.0 & 0.0 & 0.0 & 0.0 \\
\hline $\max _{i j}\left|E_{i j}^{3}\right|$ & 0.0 & 0.0 & 0.0 & 0.0 \\
\hline
\end{tabular}

TABLE 5.2. $L^{\infty}$ errors of the numerical solutions for different mesh size.
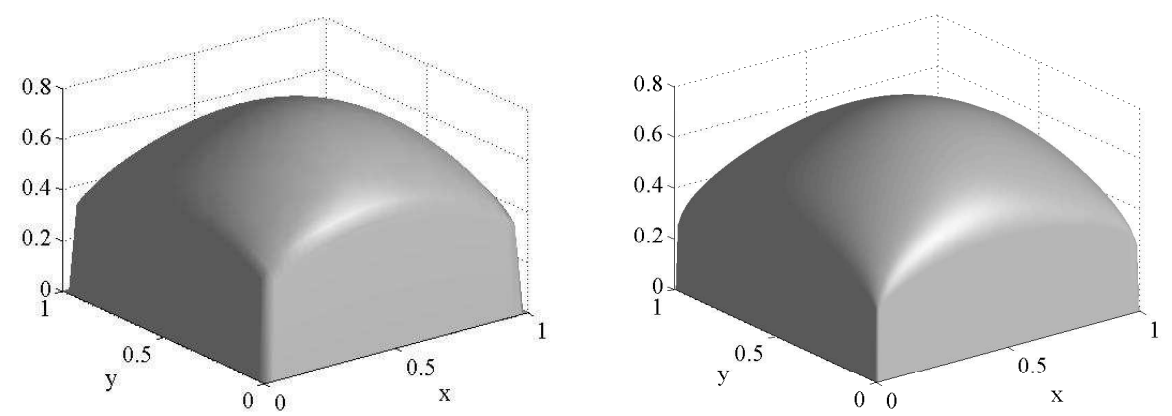

FiG. 5.2. The graphs of $u^{1}$ for Example 5.2, $\varepsilon=10^{-3}: h=\frac{1}{32}$ (left), $h=\frac{1}{128}$ (right).

\section{Conclusion}

In this paper, we present a tailored-finite-point method for a kind of linear reaction-diffusion problems in two dimensional case. First, we approximate the coefficients with piecewise constants. Then we use the eigenfunction expansion of the local reduced problem with constant coefficients to get the tailored scheme. We construct three kinds of schemes based on different functions. All of these schemes satisfy the discrete maximum principle. Furthermore, we study the error estimates of our TFPM. We prove that our TFPM can achieve good accuracy even when the mesh size $h \gg \varepsilon$. All of our numerical results support our mathematical theory.

Acknowledgement. The authors thank Prof. R. Bruce Kellogg for fruitful discussions on this work. 


\section{REFERENCES}

[1] M. Abramowitz and I.A. Stegun, Handbook of Mathematical Functions, National Bureau of Standards, 1964.

[2] A.E. Berger, H.D. Han and R.B. Kellogg, A priori estimates and analysis of a numerical method for a turning point problem, Math. Comput., 42, 465-492, 1984.

[3] I. Brayanov and I. Dimitrova, Uniformly convergent high-order schemes for a $2 D$ elliptic reaction-diffusion problem with anisotropic coefficients, Lecture Notes In Computer Science, 2542, 395-402, 2003.

[4] M. Cheng and G.R. Liu, A novel finite point method for flow simulation, Int. J. Numer. Meth. Fl., 39, 1161-1178, 2002.

[5] L.C. Evans, Partial Differential Equations, American Mathematical Society, Providence, RI, 1998.

[6] I.S. Gradshteyn and I.M. Ryzhik, Table of Integrals, Series and Products, 6th Ed., Academic Press, 2000.

[7] H. Han and Z. Huang, A tailored finite point method for the Helmholtz equation with high wave numbers in heterogeneous medium, J. Comput. Math., 26, 728-739, 2008.

[8] H. Han and Z. Huang, Tailored finite point method for a singular perturbation problem with variable coefficients in two dimensions, J. Sci. Comput. 41(2), 200-220, 2009.

[9] H. Han, Z. Huang and B. Kellogg, A Tailored finite point method for a singular perturbation problem on an unbounded domain, J. Sci. Comput., 36, 243-261, 2008.

[10] H. Han and R.B. Kellogg, Differentiability properties of solutions of the equation $-\varepsilon \Delta u+r u=$ $f(x, y)$ in a square, SIAM J. Math. Anal., 21, 394-408, 1990.

[11] Z. Huang, Tailored finite point method for the interface problem, Networks and Heterogeneous Media, 4, 91-106, 2009.

[12] A.M. Il'in, Differencing scheme for a differential equation with a small parameter affecting the highest derivative, Math. Notes, 6, 596-602, 1969.

[13] R.B. Kellogg and M. Stynes, A singularly perturbed convection-diffusion problem in a halfplane, Appl. Anal., 85, 1471-1485, 2006.

[14] J. Li and Y. Chen, Uniform convergence analysis for singularly perturbed elliptic problems with parabolic layers, Numer. Math. Theor. Meth. Appl., 1, 138-149, 2008.

[15] J. Li and I.M. Navon, Uniformly convergent finite element methods for singularly perturbed elliptic boundary value problems: convection-diffusion, Comput. Method. Appl. M., 162, 49-78, 1998.

[16] J.M. Melenk, hp-Finite Element Methods for Singular Perturbations. Lecture Notes in Mathematics, 1796. Springer-Verlag, Berlin, 2002.

[17] B. Mendez a and A. Velazquez, Finite point solver for the simulation of 2-D laminar incompressible unsteady flows, Comput. Method. Appl. M., 193, 825-848, 2004.

[18] J.J.H. Miller, On the convergence, uniformly in $\varepsilon$, of difference schemes for a two-point boundary singular perturbation problem, Numerical Analysis of Singular Perturbation Problems, P.W. Hernker and J.J.H. Millereds (eds.), Academic Press, 467-474, 1979.

[19] K.W. Morton, Numerical Solution of Convection-Diffusion Problems, Appl. Math. Math. Comput., Chapman and Hall, London, 12, 1996.

[20] K.W. Morton, M. Stynes and E. Süli, Analysis of a cell-vertex finite volume method for convection-diffusion problems, Math. Comput., 66, 1389-1406, 1997.

[21] E. Oñate. S. Idelsohn, O.C. Zienkiewicz and R.L. Taylor, A finite point method in computational mechanics. Applications to convective transport and fluid flow, Int. J. Numer. Meth. Eng., 39, 3839-3866, 1996.

[22] H.G. Roos, M. Stynes and L. Tobiska, Robust Numerical Methods for Singularly Perturbed Differential Equations, Springer, Berlin, 2nd Ed., 2008.

[23] G.I. Shishkin, A finite difference scheme on a priori adapted meshes for a singularly perturbed parabolic convection-diffusion equation, Numer. Math. Theor. Meth. Appl., 1, 214-234, 2008.

[24] M. Stynes, Steady-state convection-diffusion problems, Acta Numerica, 14, 445-508, 2005.

[25] P. Wesseling, Uniform convergence of discretization error for a singular perturbation problem, Numer. Meth. Part. D. E., 12, 657-671, 1996. 